\title{
PTERYGIUM. ITS NATURE AND A NEW LINE OF TREATMENT*
}

BY

\author{
DR. SABRI KameL
}

CAIRO

MANY theories are put forward and discussed in the literature regarding the nature of pterygium, and many papers were written on the subject, few of which tried in a precise way to describe exactly how the pterygium is formed. The aim of this paper is to report the results of a six years' investigation of the subject in a locality full of cases of pterygia. It discusses a new theory of its development, and a new line of treatment, based upon this theory, which was practised on more than 600 cases with complete success.

A true pterygium is a pathological encroachment of part of the bulbar conjunctiva exposed in the palpebral fissure over the cornea. This encroachment is in the form of a fold of the conjunctiva, triangular in shape, with the apex, called the head, on the cornea, the base merging imperceptibly in the caruncle region, or the region of the outer canthus, and the sides formed of two folds of the conjunctiva, an upper and a lower. This shape is not pathognomonic because if you pull the conjunctiva towards the cornea from any point near the limbus, it forms a triangular fold quite identical with a pterygium. The clinical appearance of the pterygium differs according to the stage in which it is seen and according to the degree of activity of the process that leads to its formation. Thus in its active and progressive stage, the fold is hyperaemic with dilated and congested radially disposed blood vessels, usually two or three in number, and the fold looks thickened, opaque and less translucent than the surrounding conjunctiva. In the meantime, surrounding the advancing head over the cornea, a halo of greyish white opacity is seen, with its convexity towards the centre of the cornea. This halo of opacity is denser near the head of the pterygium and fades away towards the centre of the cornea. It is quite superficial being present in the Epithelium and Bowman's membrane, and is, composed of greyish white dots mostly aggregated at the points where the ciliary nerves pierce Bowman's membrane. This appearance of the clinical aspect of progressive active pterygia differs according to the amount of the activity of the case. But it is always noticed that the more the hyperaemia and congestion of the conjunctival vessels, the denser is the halo of opacity in the cornea and the

\footnotetext{
* Received for publication, December 18, 1945.
} 
more progressive is the pterygium. Sooner or later, the condition begins to subside, the process being more or less self-limiting, and the regressive stage is reached in which the halo of opacity in the cornea preceding the advancing head of the pterygium stops short and begins to become absorbed and the hyperaemia and engorgement of the conjunctival vessels begins to abate. Finally the atrophic stage is reached in which no halo of opacity is seen in the cornea, the head of the pterygium thins out and becomes cicatrised, the engorgement of the conjunctival vessels disappears nearly completely and the fold of the conjunctiva in general becomes more translucent than before but still less than the surrounding normal conjunctiva.

This is the clinical description of the different stages of the development of the pterygium upon which all observers are agreed. But how and why this fold of conjunctiva encroaches upon the cornea is still an unsettled problem. It is not a problem of academic interest. On the contrary, it has great practical importance, for unless we know exactly and precisely how and why this fold encroaches upon the cornea to form the pterygium, all our treatment of the condition will be symptomatic and thus valueless. And this, I believe, is what we are doing now. Here, in Egypt, for example, the operation in vogue for the treatment of pterygium is the McReynold's tucking operation. Every pterygium we meet, we tuck. Yet in some $30-50$ per cent. of cases, we get a recurrence a few months later. In fact it is these recurrences, or failures, that drew my attention to the condition and made me start this investigation.

In a locality in which pterygium is a condition that one sees every day, all types and all stages of pterygia could be observed; compared and followed. It goes also without saying that in such a locality, with a good deal of material, so to speak, the earliest clinical signs that appear in the cases that will develop a pterygium later on could be detected.

Thus I observed in many cases that come to the hospital, a simple chronic congestion of the conjunctival blood vessels in the area exposed in the palpebral fissure, mostly nasally, but sometimes also temporally, which causes practically no discomfort, the patient coming to the hospital for something else. On observing these cases carefully, one finds an area of congestion, more intensified near the caruncle, strictly restricted to the exposed part of the bulbar conjunctiva, with few vessels running radially towards the cornea and stopping short at a varied distance from the limbus. The conjunctiva itself in such cases is slightly thickened and less translucent than the surrounding areas. When the case is severe, the vessels reach the limbus. No treatment 
was found of any avail for such conditions, and the more the patients paint their lids for their trachoma, the more the congestion of the bulbar conjunctiva in the exposed area persists. This type of congestion, thickening and loss of translucency in a restricted area of the bulbar conjunctiva exposed in the palpebral fissure is quite unknown to have any relation to trachoma, which is the most common chronic conjunctival lesion in Egypt. So I thought of examining some of these areas histo-pathologically. I selected some cases, and excised the congested area of the bulbar conjunctiva and sent it for examination.* The bare area left behind was covered by undermining the conjunctiva up and down and applying a few stitches. The result of the histo-pathological examination was identical in all cases. The epithelium is thinned, the nuclei of the submucosa have for the greater part disappeared, with hyaline and elastic tissue-like degeneration affecting the new formed connective tissue bundles in the submucosa. This pathological picture is in many aspects identical with that of pterygium. From the clinical point of view; also, if one disregards the corneal encroachment, the clinical picture of this condition is identical , with what one sees in the fold of conjunctiva that forms the pterygium. Thus the two conditions are identical pathologically, while clinically the only difference is that in one of them the affected fold of conjunctiva encroached on the corriea, and in the other it did not. This close resemblance between the two conditions could not, in my opinion, be a simple coincidence. So I followed many of these cases, which I called chronic irritative exposure conjunctivitis, for a long period at regular intervals. Some of the cases remained stationary, others subsided markedly, while the majority developed a pterygium in a period varying from three months to a year. In the latter cases, the earliest changes that are seen in the cornea are a haze at the limbus formed of minute dots, followed in time by a limbic vascularisation. The more the haze progresses towards the centre of the cornea, the more the vascularisation is attracted following it. At a certain stage of the progress, the conjunctiva begins to encroach. The whole condition is very slow taking months to develop. Thus 1 came to the conclusion that this condition that I call chronic irritative exposure conjunctivitis, must be the potential precursor of the pterygium. It does not form a pterygium on the other hand, except when certain changes do occur in the cornea. These corneal changes I presumed to be caused by the same aetiological factor that causes the conjunctival lesion.

\footnotetext{
* All the pathological specimens used in this investigation were kindly prepared and examined in the Giza Memorial Ophthalmic Laboratory. The specimens after being taken; were directly pinned with wooden pins to pieces of cork with marks showing the positions of the head and the base. They were pinned so that they would not get contracted in the preservative.
} 
Continuing the investigation further, I tried to find a cause for the encroachment itself of the conjunctiva over the cornea. Many corneal lesions, both inflammatory and degenerative, invite a limbic vascularisation, but no lesion of the cornea ever invites the encroachment of a whole fold of conjunctiva except the condition we call pterygium. The pterygium is the only lesion of the cornea in which a whole fold of conjunctiva encroaches on its surface. So continuing to take specimens and subjecting them to histo-pathological examination, I began to choose cases of well developed pterygia along with cases of very early, so to speak, budding pterygia and to compare the histo-pathological picture of both in the hope of finding a difference that may account for the encroachment of the whole fold of conjunctiva over the cornea. The result of such a comparison proved to be gratifying, for an important difference was found that can account for and explain the cause of the encroachment of conjunctiva over the cornea to form the pterygium. Thus, and to give an example of the difference I constantly found, at one and the same time I submitted four specimens to pathological examination. Two of these were of well developed pterygia while the other two were of very early pterygia. The pathological report on the well developed cases ran as follows: "The nuclei of the submucosa for the greater part have disappeared and the connective tissue bundles have become confluent with each other and look homogeneously pink (hyaline degeneration). In the deeper parts of this hyaline tissue, elastic tissue-like degeneration is'seen in the form of dark staining convoluted fibres. The epithelium over the affected area is thinned." The report on the other two cases of very early pterygia, on the other hand was "the appearances of the specimens are exactly the same as those seen in your other two specimens, but the elastic-like degenerated fibres are seen in cross section." This difference between the two pictures is in my opinion very important. For if you have horizontally disposed fibres and you cut a section through them at right angles to their axes, and you look at the section, you will see the fibres in cross section. While if the same horizontally disposed fibres shrink and contract, and you cut the same section through them, you will see the fibres in section, confluent and convoluted.

Now pterygium in my opinion, and as I will discuss later, is an inflammatory condition. Every inflammatory process heals either by complete resolution, or if the process has lasted any length of time it will lead to the damage and disappearance of the specialised elements, if any, and to a reactive overgrowth of the more resistant connective tissue cells. A replacement fibrous tissue is the response of the tissues to the effects of chronic inflammations. 
The conjunctiva and cornea are no exception to this rule. The process that leads to the formation of the pterygium is, as I said before, an inflammatory process. As a result of the deep inflammation, fibrous tissue in the form of horizontal bands is laid down. As long as the inflammatory process is restricted to the conjunctiva, no harm is done and no pterygium is formed, though the pathological changes that occur are just the same as those that one sees in the fold of conjunctiva of a well formed pterygium. But once the inflammation attacks the cornea, and the chronic conjunctivitis becomes a chronic kerato-conjunctivitis, the pterygium starts to form and the encroachment starts to occur. As a result of this kerato-conjunctivitis, fibrous tissue bands are laid down in the submucosa of both the conjunctiva and cornea. These connective tissue bands and fibres undergo the same changes that occur in fibrous tissue elsewhere. Thus in time they shrink and contract. This shrinkage will exert a certain amount of pull, and as the fibrous bands are coherent simultaneously with both the conjunctiva and cornea, this amount of pull will either pull the cornea towards the conjunctiva, or the conjunctiva towards the cornea. And as the cornea is fixed and the conjunctiva more or less mobile, this pulling effect caused by the shrinkage of the fibrous tissue bands will pull the conjunctiva, as a whole and in a triangular fold, towards the cornea in the form of what we call the pterygium. The more the case is progressive and active, the more fibrous tissue will be laid down, and the more, through its contraction, the pterygium will progress towards the centre of the cornea.

Thus in my opinion, the pterygium is the symptom of an underlying inflammatory process that starts in the bulbar conjunctiva exposed in the palpebral fissure, as a chronic irritative exposure conjunctivitis. The condition thus starting may have one of two courses to follow :-

(1) It may restrict itself to the conjunctiva alone and not affect the cornea at all, in which case you see clinically in the bulbar conjunctiva exposed in the palpebral fissure, usually in and sometimes out, a hyperaemic horizontal area extending from the caruncle towards the limbus with engorged radially disposed blood vessels which is less translucent than the surrounding area. It merges nearly imperceptibly in the healthy conjunctiva above and below. The condition has an active stage, a regressive stage and an atrophic stage just as is usually described in the clinical stages of the pterygium. Pathologically it shows the same changes seen in the fold of conjunctiva that forms the pterygium. The same thinning of the epithelium with loss of the nuclei of the submucosa, the replacement connective tissue deposition in radial 
bands and the early development of hyaline and elastic tissue-like degeneration, all of which changes seen in cases of pterygia are identically seen here. And when the condition subsides the engorgement of the vessels disappears, the translucency increases and at most a thickened part near the limbus, very much resembling a pinguecula, but more elongated and hyaline looking, is left behind.

(2) If, on the other hand, the chronic inflammation that started in the conjunctiva extends to the cornea, a pterygium is formed. The extension of the inflammatory process is by continuity and contiguity with the same aetiological factor acting on both tissues. It should be remembered that the conjunctiva and the superficial layers of the cornea are of the same embryonic origin, and it is rare for many of the inflammatory processes of the conjunctiva to heal without an affection of the superficial strata of the cornea-a fact that led Duke-Elder to view the superficial corneal affections usually accompanying acute conjunctivitis as a conjunctivitis of the cornea. And it is only when the pathological condition that starts as a conjunctivitis becomes a keratoconjunctivitis that a pterygium is formed. As a result of this kerato-conjunctivitis, fibrous tissue is laid down in the submucosa of both the conjunctiva and the cornea, the contraction and shrinkage of which will lead to the encroachment of a fold of conjunctiva over the cornea forming the pterygium. So a pterygium is neither a disease of the conjunctiva nor a disease of the corneain fact it is not a disease at all-as it is only the symptom of an underlying pathological condition that does not give the pterygium as its symptom except when it affects simultaneously the conjunctiva and the cornea.

Thus, and to sum up, a pterygium, in my opinion, is a mechanical encroachment of part of the conjunctiva over the cornea caused by the shrinkage and contraction of radially disposed fibrous tissue bundles laid down in the submucosa of the conjunctiva and in the superficial layers of the cornea, as a result of a chronic irritative exposure kerato-conjunctivitis.

\section{An aetio-pathological discussion}

Three items are worth discussion in regard to the aetiopathology of pterygium. The first is the neoplastic theory of its origin, the second is the degenerative theory of its origin, and the third is a comparison between the pterygium and pannus degenerativus.

The pterygium is supposed by many authors to be neoplastic in origin. Thus $W^{T}$ illiamson-Noble ${ }^{1}$, for example, mentions it under the heading of tumours of the eye, in Berens' book, "The 
Eye and its Diseases," though he states that its pathological nature is not clear. And John McReynolds ${ }^{2}$ in describing the surgery of the tumours of the eyeball mentions the pterygium as " one of the simple, benign primary growths on the outside of the eyeball." And Schreiter ${ }^{3}$ in 1872 considered it as a polypoid growth of the conjunctiva.

This theory of the pterygium being of neoplastic origin is not proved pathologically, and I wonder how we can agree with it, with no histo-pathological foundation. There is nothing, in fact, in the histology of the pterygium itself that suggests its being a neoplasm. And considering it to be a tumour, one would ask pterygium does not continue its activity when it is tucked down. I have never seen a pterygium when tucked down, grow down, oneself, and quite reasonably, why the "growing head" of the or in any other direction in fact. It is true that we tuck the pterygium on the fallacious presumption that if it wants to grow it might grow and extend harmlessly under the conjunctiva. But I have followed many of the cases for three and more years and not the slightest extension of the tucked head has been seen. It would only be reasonable, if the pterygium were a sort of a tumour, to expect to find some of the cases continuing their activity when tucked. There is nothing to prevent them from doing so. But according to all observations this does not happen. -And, at any rate, it would be very queer for a tumour to extend only in one direction, and only over the avascular cornea.

The theory that the pterygium is a degenerative condition, either of the conjunctiva as held by Fuchs ${ }^{4}$, Parsons $^{5}$ and others, or of the cornea as advocated by Schöninger ${ }^{6}$-is the theory in vogue nowadays, as well as being the theory most strongly advocated. The term degeneration is difficult to define accurately, but it may be said to include the visible alterations in living cells, apart from simple atrophy, which are produced by disordered nutrition and represent damage short of actual death. The disordered nutrition which leads to degeneration may be the outcome of many conditions. It may be toxic, post-inflammatory, due to the action of poisons and irritants, senile, primary . . . etc. And it is a fact that there is no hard and fast line of demarcation between a senile degeneration and, for example, a post-inflammatory one, because the cause of the degeneration bears no relation to the result. A hyaline degeneration caused by toxic nephritis is pathologically the same in consistency as a senile hyaline degeneration. It is only through the exclusion of elements of inflammation, whether chronic or acute, that we can consider the resultant hyaline degeneration to be primary in nature. Thus in the case of the pterygium the question arises whether the 
degeneration that we see in the deep layers of the conjunctiva is primary in nature, or is post-inflammatory. In my opinion, based on facts deduced from the clinical and pathological pictures, pterygium is an inflammatory lesion to begin with, while the degenerative changes seen in the deep layers of the conjunctiva are secondary and post-inflammatory in nature. Many facts are in favour of an inflammatory aetiology :

(1) The dense fibrous and connective tissue that appears in the submucosa of the conjunctiva and between Bowman's membrane and the epithelium of the cornea cannot by any means be a result of a degenerative condition. There is no known degenerative condition that leads to the formation of fibrous tissue. It is only inflammatory conditions that lead to damage and disappearance of the specialised elements, if any, and to a reactive overgrowth of the more resistant connective tissue cells. A replacement fibrous tissue is the response of the tissues to the effects of chronic inflammation, not of degeneration.

(2) The round cell infiltration of the superficial strata of the cornea and the marked increase in goblet cells in the conjunctiva which is constantly seen in pterygia, are all signs of chronic inflammation rather than of degeneration.

(3) There is no known degenerative condition that starts in a vascular well-nourished area such as the limbus, and that tends to be self-limiting the more it progresses towards the non-vascular centre of the cornea. I have seen hundreds of pterygia of different types and in different stages of development, very few of which ever reached the centre of the cornea and none at all was ever seen to extend beyond this point.

(4) The vascularity of the pterygium, the periods of more acute inflammatory engorgement that occur, the age of the patients (I saw pterygia in well-built children of 6), the occurrence in a fixed position which is the most exposed part of the bulbar conjunctiva, are all suggestive points in favour of an inflammatory aetiology.

In fact, there is nothing in favour of a degenerative aetiological theory except the finding of early signs of hyaline and elastic tissue-like degeneration in the deeper parts of the pterygium. This degeneration is a definite fact and a constant finding, but, to my way of thinking, based on facts and observations, it is also certain that this early degeneration is secondary and post-inflammatory in nature and not a primary degeneration. In fact, early degenerative changes accompany many of the chronic conjunctival inflammations. And it is not astonishing to any extent that this early degeneration occurs and goes hand in hand with many of the inflammations of a delicate and exposed tissue like the conjunctiva. A fact that led many conjunctival conditions, pinguecula 
being a marked example, to be considered as degenerative conditions, without an attempt to define whether the degeneration is primary or post-inflammatory, though it is understood that the primary type is what is meant. A pinguecula, which it should be remembered is considered by many pioneer authors as the precursor of the pterygium, is a patch of hyaline degeneration and elastic tissue proliferation in the connective tissue of the conjunctiva, supposedly due to senility and exposure. As far as senility goes, I cannot consider a pinguecula a simple senile manifestation. It is true that senility, to a certain extent, has nothing to do with age, but I cannot consider a pathological lesion present in 7 per cent. of people between the ages of $10-20$ years (according to Hinnen in 1921) a senile condition. I have seen pingueculae in children less than 10 years of age, and I have observed the development of a pinguecula in a young child of 11 years of age, 6 months after the subsiding of recurrent attacks of phlyctenular conjunctivitis. As for exposure, it is a broad term that may include many conditions, all of which lead to a single fundamental factor which is irritation, and an irritative inflammation is a known pathological entity. Thus the fact that degenerative changes, through their early onset, tend to mask the picture of a post-inflammatory degenerative condition into a primary degenerative one, should not in any way obscure our observations, which tell clearly of the true aetiological basis.

The third point in the present discussion is the comparison between the clinical condition known as degenerative pannus and the pterygium. Pannus degenerativus is a pathological condition that affects blind, shrunken globes following diseases as cyclitis, glaucoma or detachment. Clinically it starts with a dullness and unevenness of the corneal surface, with formation of vesicles, which on bursting intermittently give rise to considerable irritation. Histologically the changes arise in the substantia propria, new formed connective tissue forms which breaks through Bowman's membrane and is subsequently vascularised from the limbus. The vascularised new formed connective tissue is seen lying upon Bowman's membrane and under a thickened and degenerated epithelium. As the connective tissue under the epithelium contracts, the vessels tend to be obliterated. Subsequent changes include amyloid and hyaline degeneration and the conversion of the entire tissue into a homogeneous mass. This condition of pannus degenerativus, whether it is degenerative in nature, or whether, as I believe, it is due to the irritation of circulating toxins affecting the endothelium of the cornea, bears in its pathological picture a resemblance to that of pterygium. In both there is the same infiltration of the superficial elements 
followed by new fibrous tissue deposition. But while in pannus degenerativus, the pathological changes that occur in the cornea invite a limbic vascularisation, in the case of pterygium, a whole fold of the conjunctiva is invited to encroach. I consider this resemblance in the pathological picture and variance in the resultant sign a proof of my opinion regarding how the pterygium is formed. A pterygium does not occur if the cornea alone is affected, whatever the amount of fibrous tissue deposited, as the pannus degenerativus proves. It does not also occur if the conjunctiva alone is affected, as is seen in cases of chronic irritative exposure conjunctivitis in which the inflammation is restricted to the conjunctiva. It is only when the conjunctiva and cornea are simultaneously affected, ${ }^{-}$with the deposition of new formed subepithelial fibrous tissue bands in both, that through the contraction of this fibrous tissue, a pterygium gets formed.

\section{The aetiology}

Pterygium in my opinion is an irritative disease. The causes of the irritation are many and various, and it is not my intention here to discuss them all. I will discuss just two points that I could investigate. The first is the influence of the locality on the rate of incidence. The second is the relation between the incidence of pterygium and the patency of the lacrymal passages.

As regards the first question, I would like here to mention that the present investigation is the outcome of a 6 years' stay as the only ophthalmic surgeon in a district in Upper Egypt full of cases of pterygia, called Akhmim district. This district is a narrow strip of land, not more than 15 miles wide in its widest part, and about 40 miles long, lying between the Nile river on its western side, and the Haridi hills on its north, east and southern sides. Its population is about 120,000 and it contains 28 villages scattered here and there, some near the Nile bank and others more close to the hills. The average number of new patients seen in the only ophthalmic hospital present was about 12,000 per year, most of which were cases of conjunctival affections. Among these, pterygium was practically a daily finding. And, generally speaking, more cases of pterygia-came from the villages that were near the hills (about 75 per cent.) than those nearer to the Nile (about 25 per cent.). The greatest percentage was from two villages in particular. These are the village called Issawyia and the one called Eastern Sawamaa. The Issawyia village is near the hills and about 30 per cent. of its population work as stone cutters in the nearby stone mines, the Issawyia stone being notorious all over Upper Egypt. This might 
explain the cause of the increase of pterygium in it. The second village is an ordinary village, near the hills, with most of its population working as ordinary farmers. But it is unique in that separating it from the hills and to its northern side is a strip of sandy desert the superficial stratum of which is composed of a layer of fine sandy dust, which our northern wind carries to the village every now and then. And though there are other villages built actually on the slope of the hills, yet it was those two villages in particular that were studded with cases of pterygia. As far as the first village is concerned, the cause of the high rate of incidence of pterygium cases is obvious, for it is well known that pterygium is prevalent among stone cutters. While in the second village, I think the cause of the increase of pterygium cases in it is due to its position and the quality of the sandy dust that is blown towards it. Sandy dust is more coarse than ordinary dust and thus is more irritant, but it is not so coarse as not to be blown by winds of ordinary velocity. And just to prove the influence of the locality in this particular case, I will mention an interesting case that I observed eight years ago and am still following. It is the case of a lawyer from Sawamaa, who was practising in Akhmim (which is the principal town of the district). He came to me eight years ago to " try to do something for the reddish blush that he has got in the inner part of the white of his eyes." $\mathrm{He}$ is well built with smooth lids and emmetropic refraction. The only thing he had got was a reddish, slightly raised area extending nearly from the caruncle to the limbus in both eyes with radially disposed engorged blood vessels. It began so insidiously that he did not notice it, but it is progressing steadily though slowly. Six months later, the inflammation and redness began to attack the limbus and a very early pterygium was starting. Then he was appointed to a Governmental post in Cairo and left the Akhmim district altogether, returning only in the annual leaves. All the time no treatment of any value was given to him. After one year of his living in Cairo, I saw him and strangely enough, his progressive early pterygium could not be seen any more. The inflammation disappeared spontaneously by the simple change of the locality of habitation. And now all signs of his previous chronic irritative conjunctivitis have disappeared leaving only a comparatively thin strip of conjunctiva in the inner part of the palpebral fissure with hardly any vascularisation. This case is very convincing from many points of view, the most important of which is the marked influence of the locality of habitation on the incidence of pterygium .

The second point $I$ have tried to investigate is the relation between the patency of the lacrymal passages and the incidence of pterygium. Pterygium is an irritative condition that affects 
- the inner part of the bulbar conjunctiva more than it affects the outer part owing, most probably, to the normal flow of tears from out inwards carrying with them any dust particles or fine foreign bodies and accumulating them there and thus exciting the inflammatory process. So it would be interesting to note the relation of the percentage of lacrymal patency in cases of pterygium and otherwise. I examined 100 cases of pterygia, in different stages of development, as regards the patency of the lacrymal passages, with a control of another 100 cases, with no pterygia, taken haphazard from the out-patients. No convincing result was obtained as the percentage of non-patency was practically the same in the two groups of patients, amounting to about 14 per cent. in the former group and to 12 per cent. in the latter. So the patency of the lacrymal passages seems to bear no relation to the aetiological factors of the pterygium.

\section{Treatment}

Many operations have been devised for the treatment of pterygia, most of which are not soundly based. The operation in vogue in Egypt for the treatment of pterygium is the McReynolds' tucking operation, though in recurrent cases, other procedures are used such as diathermy coagulation of the blood vessels, transplantation, mucous grafting or total excision. The McReynolds' tucking operation is the only procedure worth discussion. The principle of the operation is to tuck the head of the pterygium, after disentangling it from the cornea, under the conjunctiva so that if the pterygium wants to "grow," it will grow and extend harmlessly. After practising this operation, even with Dr. Bakly's modification, in the treatment of my cases for a long time, I noticed that I got more than 30 per cent. recurrences. The operation is an easy and straightforward one and can be mastered even by beginners, so the high recurrence rate, which is to be considered as evidence of failure, cannot be due to a faulty technique. Many of my colleagues also reported a similar percentage of failures and some of them reported a recurrence rate as high as 50 per cent., while others reported cases that were operated upon three times and recurred a fourth time. In fact, in practically every case of progressive active pterygium, after you tuck it, a few months later you find it still well tucked down, but a new pterygium starting to form in the original site. The operation is only successful in atrophic pterygia in which the inflammation has completely subsided. Otherwise it is of no practical value, as it is only a symptomatic treatment and rarely, if ever, does a symptomatic treatment prove to be a true cure. And as a symptomatic treatment it is even of no value, because if it is only successful in atrophic cases, as in reality it is, in which the inflammation has completely sybsided, a tucking in such cases is not needed and a simple excision of the head 
would be as satisfactory and less mutilating. So from every point of view, the tucking operation is of no use or is not needed.

The treatment that $I$ have been adopting in the last six or seven years, which many of my colleagues in Egypt have adopted, has fortunately given me most satisfactory results. It is based upon the assumption that the pterygium is the symptom of an underlying, more or less self-limiting, kerato-conjunctivitis, and I have tried to imitate and enhance Nature's technique in this self-limiting process. This line of treatment has been practised on more than 600 cases and until now with complete success, a fact which proves beyond doubt the soundness of its basis. According to the assumption that the condition, basically, is a kerato-conjunctivitis, the cases may be divided into two groups : (1) Atrophic cases in which the inflammatory process has subsided completely and regression with degeneration and atrophy has taken place; (2) progressive cases in which the inflammation is still active.

For the atrophic cases, I either leave them alone, if they are very small, or operate at once. While for the progressive cases, I treat them first for about 10 to 15 days, depending upon the severity of the case, with zinc sulphate drops half per cent., 3 to 4 times daily and yellow-precipitate ointment 1 per cent. twice daily. If there is any discharge, 10 per cent. argyrol drops are given. No painting of the lids with silver nitrate is used as this causes irritation, a thing that we are trying to prevent. Generally the cases subside a good deal with this treatment, so that in most cases it need not be applied for more than 4 to 5 days. After this the patient should be operated upon.

\section{The operation}

Cocaine anaesthesia with 1 per cent. solution is used as instillation, every 2 minutes, 5 to 6 drops being sufficient. The eye is washed with a bland antiseptic solution (sublimate 1 in 7,000), and the speculum inserted. The neck of the pterygium, which is the part over the limbus, is caught with fixation forceps, and the head of the pterygium well shaved from its corneal attachment. This disentangling of the head is best done with a sharp scalpel or an old Graefe knife. This way of disentangling the head is from every point of view far better than the way in which the head is disentangled by the sawing movement of a strabismus hook passed under the neck and driven towards the centre of the cornea. After shaving the head well from the cornea, taking care not to leave adherent areas near the limbus at the upper or lower borders, the head is held with fixation forceps and the pterygium well undermined up to the caruncle with fine strabismus scissors. After undermining the whole pterygium release it and note, with the eye directed forwards, if there is any part of it redundant and lying 
over the cornea. In most cases, after freeing the head from the corneal attachment and undermining the conjunctiva to the caruncle, the fold of conjunctiva that formed the pterygium will return to its place, no upper or lower borders will be seen any more and no redundancy is noticed. Yet in some long standing cases, a small bit of the head is found to be still redundant, and this part is to be excised with the scissors so that, with the gaze directed forwards, the edge of the pterygium just abuts against the limbus. Then the pterygium is held with fixation forceps, and the fold of undermined conjunctiva raised away from the sclera and its undersurface cauterised with carbolic acid. The cauterising is done with a wooden toothpick which has been dipped in the carbolic. Only the under-surface of the conjunctiva is cauterised, care being taken not to cauterise the surface of the sclera. This finishes the operation. No stitches are needed, because there is nothing to be stitched. Before bandaging the eye operated upon, a bit of yellow-precipitate ointment 1 per cent. is put in the lower cul-desac. The patient is to be dressed daily for 4 or 5 days with zinc sulphate drops half per cent. and yellow precipitate ointment 1 per cent. After that the bandage is discarded, and for another 10 to 20 days, the zinc sulphate drops and the yellow precipitate ointment are to be continued.

The operation is very simple, though its simplicity cannot be a drawback. Its first step is to cut every connection between the fold of the conjunctiva that we call the pterygium and the cornea. This is accomplished by the close shaving of the head, and thus getting all the fibrous tissue elements on the under-surface of the head. Through this step, and the second step in which we undermine the whole area of the pterygium up to the caruncle, we ensure that all the fibrous tissue elements, which in my opinion are the cause of the conjunctival encroachment over the cornea, are sticking to the under surface of the disentangled and raised fold of conjunctiva. The third and most important step is the cauterisation of this connective tissue found now on the under-surface of the conjunctival fold. The cauterisation has a three-fold aim. It hastens the process of healing, and it hastens the complete shrinkage of the fibrous tissue bands, and through this hastening of the shrinkage, it constricts the blood vessels, cutting to a certain extent the blood supply, and thus imitating Nature's self-limiting process. For I believe that the pterygium is a self-limiting pathological condition through the diminution of the blood supply that occurs as a result of the final shrinkage and contraction of the submucous connective tissue bands and fibres. This is the aim of the cautery. And as is obvious, it is not applied so that the conjunctiva sticks to the sclera. In fact I try to obviate this as far as possible by carefully cauterising only the under-surface of the 
conjunctiva, by removing any excess of carbolic acid on the wooden applicator and by carefully swabbing any excess after cauterising. I do not as a routine cauterise the bare area of the cornea, as I found that cauterising it does not affect the end result, and cases that were non-cauterised were as successful as cauterised cases. Still cauterisation of the corneal area does not cause any harm and may be done. Immediately after the cautery, the conjunctiva shrinks a bit and a reactive hyperaemia follows. All this disappears in a few days. The zinc drops are mild astringent drops, while the yellow. precipitate ointment is a mild irritant whose property of helping the body in getting rid of replacement fibrous tissue is known, and I have found the combination of these two drugs with the operative treatment effective adjuvants in the treatment of the pterygium.

From the cosmetic point of view, the result is excellent and after a few weeks, and even before that, you can hardly see any trace of the operation. From the surgical point of view, this treatment, as proved by the successful results, is based on a perfectly sound basis. Further, I would like to mention that in the last six or seven years, about 600 cases were treated in this way, most of which were periodically followed and observed, and up till now I have not had a single recurrence.

Sufficient time has now elapsed in which to test the efficacy of this operative procedure, so that I now have every confidence in offering it to the medical profession.,

\section{REFERENCES}

1. BERENS.-The eye and its diseases. P. 314.

2. The eye and its diseases. P. 1160 .

3. DUKE-ELDER.-Text-book of ophthalmology. - Vol. II, p. 2000.

4. Text-book of ophthalmology. Vol. II, p. 2000.

5. PARsons.-Diseases of the eye. P. 183.

6. DUKE-ELdER - Text-book of ophthalmology. Vol. II, p. 2000.

\section{ANNOTATION}

\section{Sir Isaac Newton and colour vision}

The Tercentenary celebrations of the birth of Sir Isaac Newton should not be allowed to pass without an expression of the indebtedness which all students of colour vision owe to the man whom Prof. Andrade regards as the greatest scientist who has ever lived.

Not content with discovering the prismatic dispersion of light he propounded the laws of the mixture of pure colour stimuli. Of these by far the most important theoretically and practically is the fact that any colour can be matched by a suitable mixture of three selected radiations. This is the fundamental fact, and no theory of colour vision can be comprehensive which does not take it fully into 